\title{
Efecto de la Temperatura y Concentración de Tiosulfatos sobre la Velocidad de Disolución de Plata contenida en Desechos Mineros usando Soluciones $\mathrm{S}_{2} \mathrm{O}_{3}{ }^{2-}-\mathrm{O}_{2}-\mathrm{Zn}^{2+}$
}

Julio C. Juárez, Isauro Rivera, Francisco Patiño, María I. Reyes

Universidad Autónoma del Estado de Hidalgo, Área Académica de Ciencias de la Tierra y Materiales, Carretera Pachuca-Tulancingo Km. 4.5, C.P. 42184 Mineral de la Reforma, Hidalgo-México. (e-mail: jutj731101@hotmail.com)

Recibido Nov. 07, 2011; Aceptado Ene. 06, 2012; Versión final recibida Ene. 18, 2012

\section{Resumen}

Se estudió el efecto de la temperatura y la concentración de tiosulfatos sobre la velocidad de disolución de la plata contenida en desechos mineros, utilizando el sistema $\mathrm{S}_{2} \mathrm{O}_{3}{ }^{2-}-\mathrm{O}_{2}-\mathrm{Zn}^{2}$. En el Estado de Hidalgo en México hay miles de toneladas de desechos mineros con contenidos de plata del orden de 71 gramos de plata por tonelada de desecho, razón que motiva el estudio. Se observó que la temperatura influye drásticamente sobre la velocidad de lixiviación de la plata, presentando una energía de activación $\mathrm{Ea}=55.85 \mathrm{~kJ} \mathrm{~mol}^{-1}$, indicando que la reacción de disolución está controlada por la reacción química. La concentración de tiosulfatos presenta una considerable influencia sobre la velocidad de disolución del metal precioso. La máxima disolución de plata fue de aproximadamente $97 \%$ a $45^{\circ} \mathrm{C}$ y $40 \mathrm{gL}^{-1}$ de $\left[\mathrm{S}_{2} \mathrm{O}_{3}{ }^{2-}\right]$. Se concluye que el proceso estudiado parece una buena alternativa a otros procesos como la tóxica cianuración.

\section{Temperature and Concentration Effect of Thiosulfates on the Dissolution Rate of Silver contained in Mining Waste using $\mathrm{S}_{2} \mathrm{O}_{3}{ }^{2-}-\mathrm{O}_{2}-\mathrm{Zn}^{2+}$ Solutions}

\begin{abstract}
The effect of the temperature and thiosulfate concentration on the dissolution rate of silver contained in mining wastes, using the system $\mathrm{S}_{2} \mathrm{O}_{3}{ }^{2-}-\mathrm{O}_{2}-\mathrm{Zn}^{2+}$ was studied. In the State of Hidalgo in México, thousands of tons of waste mining having around 71 grams of silver per ton of waste have been accumulated over the years. This is the main reason that motivates this study. It was observed that the temperature drastically affects the leaching rate of the silver showing an activation energy $\mathrm{Ea}=55.85 \mathrm{~kJ} \mathrm{~mol}^{-1}$, implying that the dissolution reaction is controlled by the chemical reaction. The thiosulfate concentration shows a considerable influence on the precious metal dissolution rate. The maximum silver dissolution was found to be approximately $97 \%$ at 45 ${ }^{\circ} \mathrm{C}$ and $40 \mathrm{gL}^{-1}$ of $\left[\mathrm{S}_{2} \mathrm{O}_{3}{ }^{2-}\right]$. It is concluded that the process studied represents a good alternative when compared to other processes such as the toxic cyanidation reaction.
\end{abstract}

Keywords: leaching, tailings, silver, thiosulfates 


\section{INTRODUCCIÓN}

La plata forma parte importante en la economía de México, debido a que es uno de los principales productores de este metal precioso a nivel mundial, por más de cien años el proceso de cianuración ha sido utilizado para la extracción de valores metálicos como el oro y la plata a partir de sus minerales, sin embargo una de las desventajas que tiene este proceso es su alto grado de toxicidad, además que los minerales refractarios no pueden ser tratados por este proceso convencional debido a que se requieren altos consumos de cianuro y las recuperaciones son muy limitadas. Esta es una de las razones para buscar alternativas de reactivos lixiviantes, el sistema basado en la lixiviación con tiosulfatos $\left(\mathrm{S}_{2} \mathrm{O}_{3}{ }^{2-}\right)$ es considerado en la actualidad una alternativa no toxica al proceso de cianuración (Jeffrey et al., 2002, Rivera 2003 y Muir et al. 2005) y como medio de disolución de minerales refractarios (Schmitz et al., 2001) ya que una de las ventajas del proceso con respecto al cianuro es la selectividad en la extracción de plata contenida en fases refractarias. El interés de buscar alternativas al cianuro como reactivo lixiviante es principalmente, intentar aumentar el grado de disolución de metales preciosos contenidos en minerales refractarios, Pocos estudios se han llevado a cabo con tiosulfatos debido a la mineralogía y a la complejidad de la química de la solución (Breuer et al., 2000, Jeffrey 2001 y Feng et al., 2007). De las primeras investigaciones que se tienen reportadas es la que realizaron (Zipperian et al., 1988) quienes estudiaron la disolución de oro y plata de minerales de riolita, alcanzando una extracción de plata del $60 \%$ aproximadamente para tiempos de lixiviación de 3 horas y con concentraciones de tiosulfatos del $42 \%$, la temperatura influye dramáticamente en la extracción de plata, incrementándose desde $18 \%$ para temperatura del orden de $25^{\circ} \mathrm{C}$, a extracciones del $60 \%$ para $60^{\circ} \mathrm{C}$., (Kuzugüdenli, O. E. and Kantar, C. 1999) reportan que los tiosulfatos son una buena alternativa para la lixiviación del oro, mencionan que el proceso se ve favorecido con la presencia de iones de cobre y que la temperatura y la concentración de oxígeno disuelto, son los principales factores que afectan la velocidad de disolución del metal. (Jeffrey et al., 2000), llevaron a cabo la lixiviación de oro y plata utilizando una microbalanza rotatoria electroquímica de cristal de cuarzo; en el estudio, se menciona que la velocidad de disolución esta limitada por la difusión del ion cúprico. Se calcula también, el coeficiente de difusión de acuerdo a la ley de Levich, encontrando que la reacción está controlada por difusión en película. (McNulty, T. 2001) realizó la investigación de cinco sistemas diferentes, para encontrar agentes lixiviantes alternativos al cianuro, para la lixiviación de oro y plata, reportando que no existe ninguna razón convincente para que estos sistemas no alcancen el porcentaje de oro disuelto comparado con el proceso de cianuración. Recientemente, (Hernández, 2009), estudia la recuperación de Ag contenida en escombreras previamente remolidas en medio tiosulfatos, sin el uso de catalizadores de reacción, alcanzando recuperaciones del $66 \%$ a $25^{\circ} \mathrm{C}$, en un tiempo de 4 horas.

El presente estudio, se desarrolló con el objetivo de optimizar el proceso de lixiviación de plata en medio $\mathrm{O}_{2}-\mathrm{S}_{2} \mathrm{O}_{3}{ }^{2-}$ con la adición de $\mathrm{Zn}^{2+}$ como catalizador, en un rango de temperaturas establecido, para alcanzar las máximas disoluciones de plata en tiempos más cortos.

\section{PROCEDIMIENTO EXPERIMENTAL}

Para desarrollar esta investigación se utilizaron los jales de dos Carlos, del Estado de Hidalgo, México, debido a que ésta área cuenta con un volumen total de 14.3 millones de toneladas métricas. Las muestras extraídas en campo fueron homogenizadas para caracterizarlas química y mineralógicamente, haciendo uso de las técnicas de Difracción de Rayos $X$ (DRX), Fluorescencia de Rayos X (FRX), Espectroscopia de emisión por plasma acoplado por inducción (ICP) y Copelación. Los experimentos de lixiviación se llevaron a cabo en un reactor de vidrio convencional de $500 \mathrm{~mL}$ de capacidad, montado en una parrilla con medidor de la velocidad de agitación y controlador de temperatura.

Se implementó un sistema de medición continuo de $\mathrm{pH}$ basado en un $\mathrm{pH}$-metro, equipado con un electrodo de $\mathrm{pH}$ apto para operar en condiciones extremas de acidez y alcalinidad (rango de $\mathrm{pH}$ de 0-14). Los ajustes de $\mathrm{pH}$ de los experimentos, se realizaron adicionando una solución $\mathrm{H}_{2} \mathrm{SO}_{4}$ al $1 \%$ directamente al reactor por medio de una bureta graduada. Se inyecto oxígeno para dar una atm de presión en el interior del reactor. La temperatura del sistema fue controlada por medio de 
un termopar acoplado a la parrilla de calentamiento. El inicio de la reacción se tomó como aquel instante en que la muestra del mineral entra en contacto con la solución lixiviante. Se tomaron muestras a diferentes tiempos t. Las condiciones experimentales utilizadas en el presente estudio cinético se presentan en la Tabla 1.

Tabla 1: Condiciones experimentales del estudio.

\begin{tabular}{cc}
\hline Parámetros & Condiciones Experimentales \\
\hline Diámetro de Partícula (micras) & 106 \\
{$\left[\mathrm{Zn}^{2+}\right]\left(\mathrm{g} \mathrm{L}^{-1}\right)$} & 4.4 \\
{$\left[\mathrm{~S}_{2} \mathrm{O}_{3}{ }^{2-}\right]\left(\mathrm{g} \mathrm{L}^{-1}\right)$} & $5,10,20,40,80$ y 100 \\
Temperatura $\left({ }^{\circ} \mathrm{C}\right)$ & $25,30,35,40 \mathrm{y} 45$ \\
Velocidad de Agitación $\left(\mathrm{min}^{-1}\right)$ & 750 \\
Sistema de agitación & Magnético \\
Presión Parcial de $\mathrm{O}_{2}(\mathrm{~atm})$ & 1.0 \\
$\mathrm{pH}$ & 6 \\
\hline
\end{tabular}

Las soluciones de muestras extraídas en los experimentos de lixiviación, fueron analizadas por Espectrofotometría de Absorción Atómica (EAA) para determinar la concentración de plata en solución al tiempo t.

La fracción de plata fue calculada de acuerdo a la siguiente expresión:

$$
X s=\frac{A g_{s o l}}{A g_{T}}
$$

Donde: $X_{S}=$ Fracción de $\mathrm{Ag}$ en solución, $[\mathrm{Ag}]_{\mathrm{sol}}=$ Concentración de $\mathrm{Ag}$ en un tiempo $\mathrm{t},[\mathrm{Ag}]_{\mathrm{T}}=$ Concentración de plata total.

\section{RESULTADOS Y DISCUSIÓN}

\section{Caracterización del mineral}

Los resultados obtenidos de las técnicas de caracterización mencionadas en la metodología confirman que la composición química del mineral de inicio, está compuesta en su mayoría por $\mathrm{SiO}_{2}(75 \%)$, seguido de $\mathrm{Al}_{2} \mathrm{O}_{3}(8 \%), \mathrm{Fe}_{2} \mathrm{O}_{3}(5 \%), \mathrm{SO}_{3}(5 \%), \mathrm{K}_{2} \mathrm{O}(3.4 \%), \mathrm{CaO}(1.5 \%), \mathrm{Na}_{2} \mathrm{O}$ (0.4\%), $\mathrm{MgO}(0.4 \%) \mathrm{TiO}_{2}(0.3 \%), \mathrm{MnO}(0.15 \%)$ y $\mathrm{P}_{2} \mathrm{O}_{5}(0.1 \%)$, mientras que la concentración del metal precioso plata se encuentra en el orden de $71 \mathrm{~g} / \mathrm{ton}^{-1}$.

\section{Efecto concentración de tiosulfatos}

Para conocer el efecto del agente complejante sobre el grado de disolución de la plata, se realizaron varios experimentos a diferentes concentraciones de tiosulfatos, la representación gráfica de los valores obtenidos en la lixiviación de $\mathrm{Ag}^{+}$se presenta en la Figura 1, en la cual se observa que para la menor concentración de tiosulfatos se obtiene una recuperación del 48 \%, la cual va incrementando al aumentar la concentración del tiosulfatos, hasta alcanzar un máximo de liberación de plata en solución $(69.4 \%)$ al utilizar $40 \mathrm{gL}^{-1}$ de tiosulfatos; sin embargo, para concentraciones altas de tiosulfatos la disolución del metal precioso es del $64.9 \%$.

Las constantes experimentales de velocidad $\left(K_{\exp }\right)$ fueron calculadas mediante regresión lineal a partir de las curvas de [Ag] en solución frente al tiempo, de los experimentos de lixiviación, el valor de la pendiente representa la $\mathrm{K}_{\exp }$. El orden de reacción (n) se determinó por regresión lineal graficando el $\log \left[\mathrm{S}_{2} \mathrm{O}_{3}{ }^{2-}\right]$ frente al log de las $\mathrm{K}_{\text {exp }}$, para obtener la línea recta de pendiente $\mathrm{m}$, que nos indica el valor de $n$ el cual se establece en $n=0.39$ en el rango de 5 a $20 \mathrm{gL}^{-1}$ y de $n=0$ en el rango de 20 a $100 \mathrm{gL}^{-1}$, como se puede observar existe un punto de saturación del agente complejante para la lixiviación de plata, el cual se puede observar en la Figura 2. 


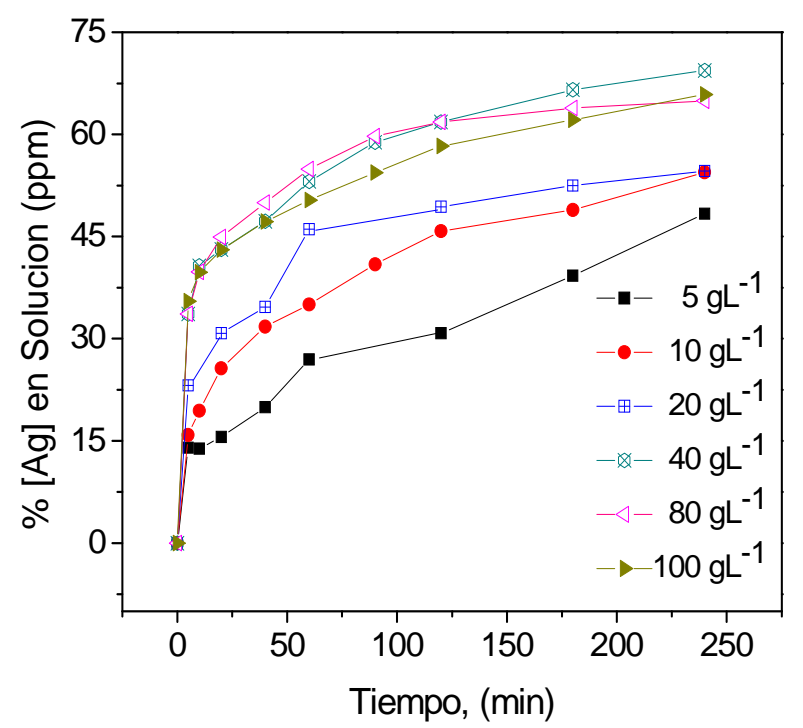

Fig.1: Porcentaje de $[\mathrm{Ag}]$ en solución en función del tiempo a diferentes concentraciones de tiosulfatos.

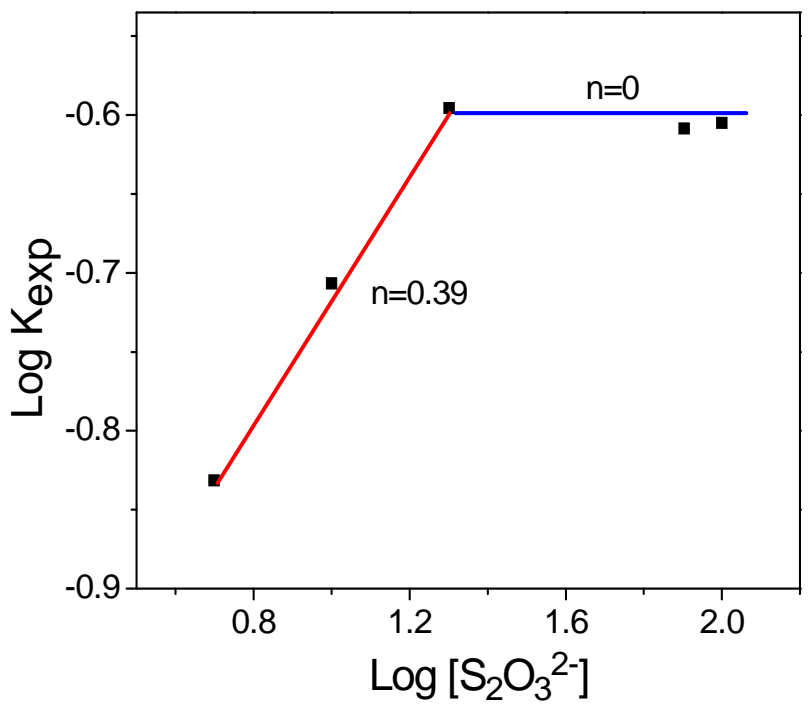

Fig. 2: Dependencia de la $\mathrm{K}_{\exp }$ frente a la $\left[\mathrm{S}_{2} \mathrm{O}_{3}{ }^{2-}\right]$ Orden de reacción $\mathrm{n}=0.39$, en el rango de 5 a $20 \mathrm{gL}^{-1}$.

\section{Efecto Temperatura}

Otra variable muy importante en este trabajo fue la temperatura, en la Figura 3 se representan los valores obtenidos de los experimentos en el rango de temperaturas evaluado. En la cual se puede observar, tiempos de ataque de 240 minutos y que a $25{ }^{\circ} \mathrm{C}$ la recuperación de plata en solución es del $54.6 \%$, mientras que para $45^{\circ} \mathrm{C}$ se incrementa drásticamente ésta recuperación, hasta alcanzar un $97.13 \%$ de plata en solución, es decir hay un aumento muy significativo de $\mathrm{Ag}$ en la solución lixiviante al incrementar la temperatura en el proceso. Estos resultados son consistentes con lo reportado por (Zipperian et al., 1988) y (Kuzugüdenli, O. E. and Kantar, C., 1999); sin embargo, en ésta investigación se obtiene mayor porcentaje de disolución del metal precioso.

El parámetro cinético para determinar el tipo de control de una reacción química es la energía de activación (Ea), la cual está relacionada a la ecuación de Arrhenius de acuerdo a la siguiente ecuación. 


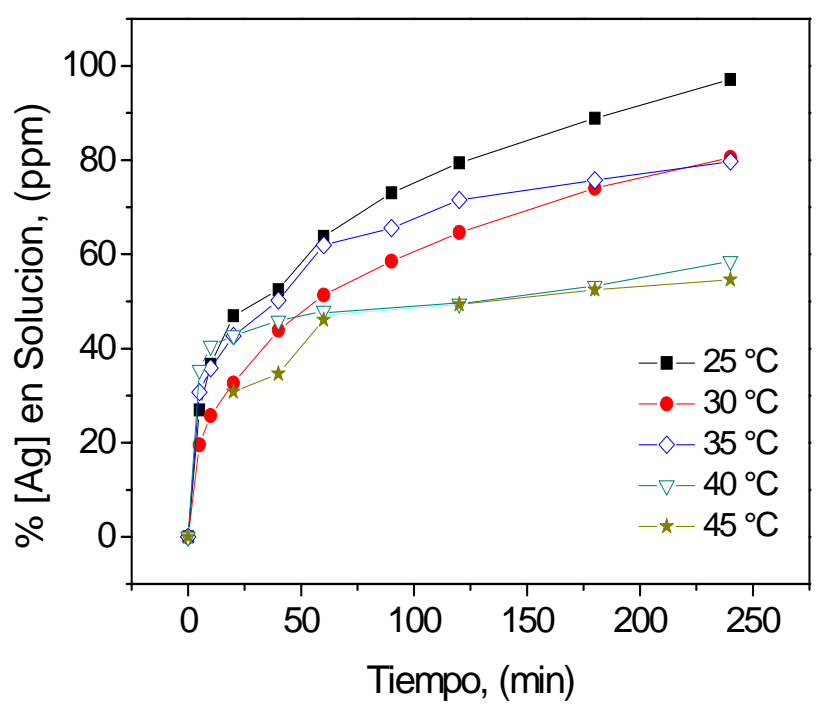

Fig. 3: Porcentaje de [Ag] en solución en función del tiempo, a diferentes temperaturas.

Para calcular la energía de activación del sistema, se grafican los valores que se presentan en la Tabla 2 del logaritmo neperiano de $K_{\text {exp }}$ en función del recíproco de la temperatura, en donde la pendiente del ajuste lineal de los datos experimentales representa la energía de activación (Ea) dividida por el valor negativo de la constante universal de los gases (R), como se muestra en la Figura 4.

Tabla 2: Dependencia de la Kexp en función de la temperatura.

\begin{tabular}{ccccc}
\hline $\mathrm{T}\left({ }^{\circ} \mathrm{C}\right)$ & $\mathrm{T}(\mathrm{K})$ & $1000 / \mathrm{T}^{-1}\left(\mathrm{~K}^{-1}\right)$ & $\mathrm{K}_{\exp }\left(\mathrm{min}^{-1}\right)$ & $\mathrm{In} \mathrm{K}_{\exp .}\left(\mathrm{min}^{-1}\right)$ \\
\hline 25 & 298 & 3.355704 & 0.0005 & -7.60090 \\
30 & 303 & 3.300330 & 0.0006 & -7.41858 \\
35 & 308 & 3.246753 & 0.0010 & -6.90775 \\
40 & 313 & 3.194888 & 0.0016 & -6.43775 \\
45 & 318 & 3.144654 & 0.0018 & -6.31996 \\
\hline
\end{tabular}

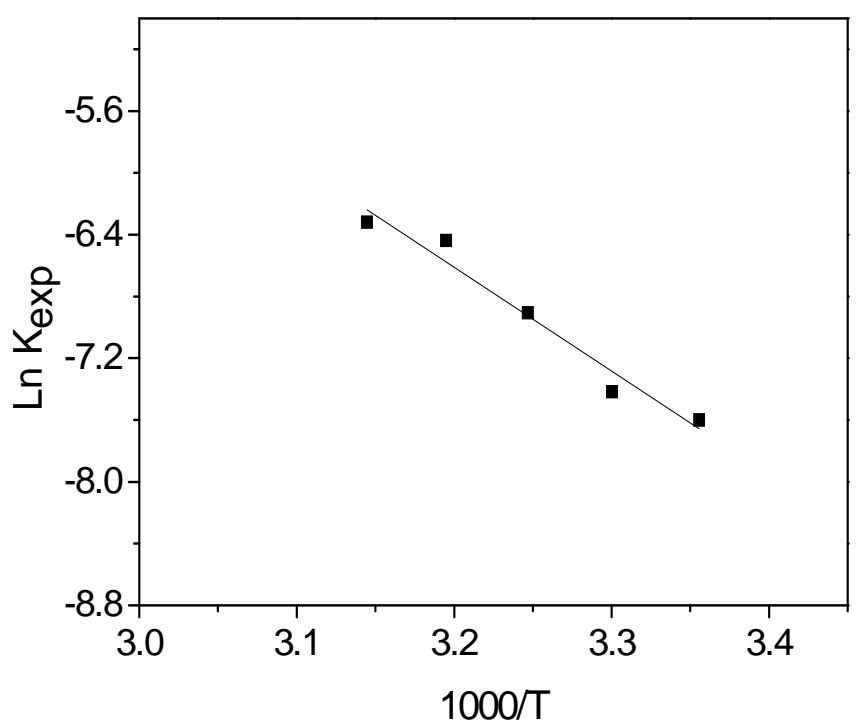

Fig. 4: Dependencia de la Kexp en función de la temperatura.

Energía de activación $\mathrm{Ea}=55.85 \mathrm{~kJ} \mathrm{~mol}^{-1}$

En esta Figura se representan gráficamente las constantes experimentales (In de $K_{\text {exp }}$ ) frente a la variación de la temperatura $(1000 / T)$, donde se obtiene una pendiente negativa, la cual nos permite calcular la energía de activación del sistema, que para este caso fue establecida en 55.85 
$\mathrm{kJ} \mathrm{mol}^{-1}$, este valor es indicativo del claro efecto de la temperatura sobre la velocidad global de la reacción. Es bien conocido que valores superiores a $40 \mathrm{~kJ} \mathrm{~mol}^{-1}$ de energía de activación corresponden a procesos controlados por la propia reacción química.

\section{CONCLUSIONES}

En el proceso de lixiviación de plata propuesto, se observó que al incrementar la concentración de tiosulfatos, aumenta la plata disuelta en la solución lixiviante, siendo del $48.35 \%$ al utilizar una concentración de $5 \mathrm{gL}^{-1}$, hasta $69.4 \%$ para una concentración de $40 \mathrm{gL}^{-1}$ a temperatura ambiente. $\mathrm{El}$ orden de reacción frente a la $\left[\mathrm{S}_{2} \mathrm{O}_{3}{ }^{2-}\right]$ fue de $\mathrm{n}=0.39$ en el rango de 5 a $20 \mathrm{gL}^{-1}$. La temperatura tiene una marcada influencia sobre el grado de disolución de la plata. La energía de activación del sistema es de $55.85 \mathrm{~kJ} \mathrm{~mol}^{-1}$, indicativo que el proceso está controlado por la reacción química. Así mismo, al incrementar la temperatura se elevó la recuperación del metal precioso, obteniendo recuperaciones del $54.6 \%$ a $25^{\circ} \mathrm{C}$, incrementándose hasta el $97.13 \%$ a $45{ }^{\circ} \mathrm{C}$, con $20 \mathrm{gL}^{-1}$ de $\left[\mathrm{S}_{2} \mathrm{O}_{3}{ }^{2-}\right]$. Además que el proceso de disolución de plata se ve favorecido por la presencia de iones de $\mathrm{Zn}^{2+}$. Por lo tanto el sistema propuesto puede ser una buena alternativa para recuperar la plata contenida en residuos mineros.

\section{REFERENCIAS}

Ballester, A., Verdeja, L.F. y Sancho, J., Metalurgia Extractiva, Fundamentos, vol. 1, Editorial Síntesis, España, (2000).

Breuer P. L. y Jeffrey M. I., Thiosulfate leaching kinetics of gold in the presence of cupper an ammonia, Hydrometallurgy, 442-452, (2000).

D. Zipperian, S. Raghavan, J.P. Wilson. Gold and silver extraction by ammoniacal thiosulfate leaching from a rhyolite ore. Hydrometallurgy, 19-361, (1988).

Feng D. y Van Deventer J. S., The role of oxygen in thiosulfate leaching of gold, Hydrometallurgy, 85, 193-202, (2007).

Hernández A. J., Cinética de molienda y recuperación de Ag mediante procesos convencionales y no convencionales de las escombreras de la industria minero-metalúrgica del Estado de Hidalgo, Tesis de Doctor en Ciencias, AACTyM, UAEH, México, (2009).

Jeffrey M.I., Kinetic aspects of gold and silver leaching in ammonia-thiosulfate solutions. Hydrometallurgy, 60, 7-16, (2001).

Jeffrey M. I., Breuer P. L. W. L. y Choo W. L., A kinetic study that compares the leaching of gold in the cyanide, thiosulfate and chloride system, Metallurgical and. Materials Transactions B, 32B, 979-986, (2002).

Kuzugüdenli, O. E., Kantar, C. Alternatives to gold recovery by cyanide leaching, Erc. Univ. Fen Bil. Derg. 15, 1-2, 119-127, (1999).

Levenspiel, O., Ingeniería de las reacciones químicas, Ed. Reverte, Barcelona, (2000).

McNulty, T., Cyanide substitutes. Mining Magazine, 184-5, 256-261, (2001).

Muir D. M. y Aylmore M. G., Thiosulfate as an alternative to cyanide for gold processing-Issues and impediments, Miner. Process, 15, Elsevier, Sydney, 113, (2005).

Rivera I., Estudio cinético de la precipitación/Lixiviación de la plata en el sistema $\mathrm{O}_{2}-\mathrm{S}_{2} \mathrm{O}_{3}{ }^{2-}-\mathrm{S}_{2} \mathrm{O}_{4}{ }^{2-}$, PhD Thesis, Universitat de Barcelona, (2003).

Schmitz P.A. Duyvesteyn S., Johnson W.P., Enloe L. y McMullen J. Ammoniacal thiosulfate and sodium cyanide leaching of preg-robbing gold strike ore carbonaceous matter, Hydrometallurgy, 60, 2540, (2001). 